\title{
Effect of halloysite nanoclay concentration and addition of glycerol on mechanical properties of bionanocomposite films
}

\begin{abstract}
Conventional plastic made from petrochemical based polymer which is non-degradable is not environmentally friendly. Thus, there is a need to develop bionanocomposite films to replace the non-degradable plastic. Bionanocomposite films were produced by the casting method using polylactic acid (PLA) biopolymer with the incorporation of nanoclay, particularly halloysite. The effect of the halloysite nanoclay concentration $(0,2,4,6$ and $8 \mathrm{wt} . \%)$ as well as the addition of a plasticiser, particularly glycerol, on the mechanical properties (tensile stress, elongation at break and tensile strength) of bionanocomposite films was investigated. It was found that without the addition of glycerol, 2 wt.\% concentration of halloysite nanoclay film resulted in the maximum tensile strength and elongation at break due to the interaction of the PLA and the nanoclay which is known for interfacial adhesion. The interaction was confirmed by FTIR spectrum. When more than $2 \mathrm{wt} . \%$ of nanoclay clay was added, both the tensile strength and the elongation at break reduced due to the agglomeration of the nanoclay. However, when glycerol was added, the film without the addition of halloysite nanoclay exhibited the maximum tensile strength and the film with 8 wt.\% halloysite nanoclay exhibited the maximum elongation at break. The addition of glycerol lowered the mechanical properties of the films because the intercalation of the glycerol into the clays prevented the entry of PLA into the interlamellar of the nanoclay.
\end{abstract}

Keyword: Bionanocomposite; Glycerol; Nanoclay; Mechanical properties; Polylactic acid; FTIR 